\title{
Sensitivity of simulated climate to conservation of momentum in gravity wave drag parameterization
}

Article

Published Version

Shaw, T. A., Sigmond, M., Shepherd, T. G. and Scinocca, J. F. (2009) Sensitivity of simulated climate to conservation of momentum in gravity wave drag parameterization. Journal of Climate, 22 (10). pp. 2726-2742. ISSN 1520-0442 doi: https://doi.org/10.1175/2009JCLI2688.1 Available at https://centaur.reading.ac.uk/31769/

It is advisable to refer to the publisher's version if you intend to cite from the work. See Guidance on citing.

To link to this article DOI: http://dx.doi.org/10.1175/2009JCLI2688.1

Publisher: American Meteorological Society

All outputs in CentAUR are protected by Intellectual Property Rights law, including copyright law. Copyright and IPR is retained by the creators or other copyright holders. Terms and conditions for use of this material are defined in the End User Agreement.

www.reading.ac.uk/centaur 
Central Archive at the University of Reading

Reading's research outputs online 


\title{
Sensitivity of Simulated Climate to Conservation of Momentum in Gravity Wave Drag Parameterization
}

\author{
Tiffany A. Shaw, Michael Sigmond, and Theodore G. Shepherd \\ Department of Physics, University of Toronto, Toronto, Ontario, Canada \\ JOHN F. SCINOCCA \\ Canadian Centre for Climate Modelling and Analysis, University of Victoria, Victoria, British Columbia, Canada
}

(Manuscript received 23 June 2008, in final form 8 February 2009)

\begin{abstract}
The Canadian Middle Atmosphere Model is used to examine the sensitivity of simulated climate to conservation of momentum in gravity wave drag parameterization. Momentum conservation requires that the parameterized gravity wave momentum flux at the top of the model be zero and corresponds to the physical boundary condition of no momentum flux at the top of the atmosphere. Allowing momentum flux to escape the model domain violates momentum conservation. Here the impact of momentum conservation in two sets of model simulations is investigated.

In the first set, the simulation of present-day climate for two model-lid height configurations, 0.001 and 10 $\mathrm{hPa}$, which are identical below $10 \mathrm{hPa}$, is considered. The impact of momentum conservation on the climate with the model lid at $0.001 \mathrm{hPa}$ is minimal, which is expected because of the small amount of gravity wave momentum flux reaching $0.001 \mathrm{hPa}$. When the lid is lowered to $10 \mathrm{hPa}$ and momentum is conserved, there is only a modest impact on the climate in the Northern Hemisphere; however, the Southern Hemisphere climate is more adversely affected by the deflection of resolved waves near the model lid. When momentum is not conserved in the 10 -hPa model the climate is further degraded in both hemispheres, particularly in winter at high latitudes, and the impact of momentum conservation extends all the way to the surface.

In the second set of simulations, the impact of momentum conservation and model-lid height on the modeled response to ozone depletion in the Southern Hemisphere is considered, and it is found that the response can display significant sensitivity to both factors. In particular, both the lower-stratospheric polar temperature and surface responses are significantly altered when the lid is lowered, with the effect being most severe when momentum is not conserved. The implications with regard to the current round of Intergovernmental Panel on Climate Change model projections are discussed.
\end{abstract}

\section{Introduction}

The robustness of the modeled response to climate change (e.g., from increases in carbon dioxide and ozone depletion) is an important issue because robustness increases confidence in climate change predictions. Parameterizations of unresolved processes are a key part of this because they arguably represent the largest uncertainty in climate modeling. The fact that parameterizations can be tuned to obtain the current climate does not necessarily imply that they will respond cor-

Corresponding author address: Dr. Theodore G. Shepherd, Department of Physics, University of Toronto, 60 St. George St., Toronto, ON M5S 1A7, Canada.

E-mail: tgs@atmosp.physics.utoronto.ca rectly to climate perturbations; there is a need to ensure that their responses to climate perturbations are physical. Unphysical sensitivities and feedbacks from parameterizations need to be identified and minimized.

In the case of the parameterization of gravity wave drag (GWD), Shepherd and Shaw (2004) argued that momentum conservation is a key physical constraint, and that nonconservation can lead to spurious downward influence from a middle-atmospheric radiative perturbation. In the context of GWD parameterization, momentum conservation requires that any gravity wave momentum flux through a vertical model level must be entirely absorbed in the atmosphere above (i.e., there can be no radiation of momentum flux to space). Thus, the amount of momentum flux at the model lid can be used as a measure of conservation; zero momentum flux 
at the model lid implies that momentum is conserved. However, the general practice in climate modeling has been to let momentum flux escape to space. Shaw and Shepherd (2007, hereafter SS07) examined the impact of momentum conservation in a two-dimensional framework, focusing on the response of nonorographic GWD to idealized polar ozone depletion. SS07 showed that the modeled response was robust to changes in the model-lid height, gravity wave source spectrum, and choice of parameterization scheme if momentum was conserved, but exhibited strong sensitivities to these choices when momentum was not conserved. This result has implications for the practical question of how high model lids should be in realistic climate models with resolved large-scale waves. To address this question it is necessary to compare simulations with high- and low-lid models. The results of SS07 suggest momentum conservation in parameterized GWD is essential to obtain a well-posed comparison; SS07 argued that the lack of momentum conservation explained earlier quite dramatic results of Lawrence (1997).

An obvious next step is to quantify the importance of momentum conservation in a more realistic context, namely, a general circulation model (GCM). This is the purpose of the present study. In addition to quantifying the importance of momentum conservation in a realistic setup with three-dimensional winds and orographic GWD (not examined by SS07), the indirect effect of dynamical feedbacks via changes in the Eliassen-Palm flux divergence (EPFD; e.g., McLandress and McFarlane 1993) can also be examined. Both orographic GWD and feedbacks on EPFD offer the potential of a much stronger impact of momentum conservation on surface climate than would be expected from nonorographic GWD alone. It is important to understand how these dynamical effects of momentum conservation affect both the present-day climate and its response to climate perturbations.

Section 2 discusses the model configurations and simulations. Because the largest sensitivities are found near the poles, the results focus on the response at high latitudes. Section 3 discusses the impact of momentum conservation on the modeled control climate, while the impact of momentum conservation on the modeled response to idealized ozone depletion is considered in section 4 . The paper concludes with a summary and discussion in section 5 .

\section{Model configuration}

The GCM used in this study is the Canadian Middle Atmosphere Model (CMAM) (Scinocca et al. 2008). The version used here is the same as that used for the 2006 World Meteorological Organization Ozone Assessment (Eyring et al. 2006), except in this study the interactive chemistry is turned off. In its standard configuration the model has 71 vertical levels from the surface up to $0.001 \mathrm{hPa}(\sim 100 \mathrm{~km})$, and both the mean fields and their variability have been shown to agree well with observations in the extratropical latitudes of both hemispheres (Scinocca et al. 2008; McLandress and Shepherd 2009; Hitchcock et al. 2009). The orographic and nonorographic GWD are parameterized according to Scinocca and McFarlane (2000) and Scinocca (2003), respectively. Here we will refer to the standard configuration as the high configuration. The other model configuration used, referred to as the lowered configuration, is the same as the standard configuration, except with all model levels above $10 \mathrm{hPa}$ eliminated (Sigmond et al. 2008). The lowered configuration has 41 vertical levels up to $10 \mathrm{hPa}$ and has an identical time step, resolution (both horizontal and vertical), and parameterized physics, including the orographic and nonorographic GWD settings, as that of the high configuration. However, the nonzonal sponge layer that is applied in the high configuration near the model lid is not applied in the lowered configuration. It is important that the lowered configuration preserve the aspects of the high configuration below $10 \mathrm{hPa}$ so that a comparison between the two configurations is not ill posed (Sigmond et al. 2008).

Two sets of four model simulations were run, each for $40 \mathrm{yr}$. Each set involved running the Canadian Middle Atmosphere Model (CMAM) with and without conservation of parameterized momentum flux for the two model-lid height configurations. The first set of four simulations, discussed in section 3, are labeled as high configuration, conservative (HIGH_C); high configuration, nonconservative (HIGH_N); lowered configuration, conservative (LOW_C); and lowered configuration, nonconservative (LOW_N) and are used to quantify the impact of momentum conservation on the control (present day) climate. The second set of four simulations, discussed in section 4 , is identical to the first, except that a perturbation is added to the specified ozone, which is designed to mimic Southern Hemisphere springtime polar ozone depletion. Simulations in this second set are labeled following the conventions of the first set, but are prefaced by "O3" (e.g., O3_HIGH_C indicating high configuration, conservative, and with the ozone perturbation). In this analysis the first set of simulations is taken as the control for the second set, which allows us to quantify the impact of model-lid height and momentum conservation on the response to ozone depletion.

Conservation of parameterized momentum flux was enforced in both lid height configurations by depositing 
the momentum flux at the model lid in the uppermost model level (ensuring zero flux at the lid). This procedure is a proxy for the net GWD that would occur above this level, and acts to represent the upwelling or downwelling below the lid that would be induced by GWD above the lid. It ensures that the integral of GWD from the launch level to the lid $\left(z_{T}\right)$ equals the total momentum flux introduced at the launch level, that is,

$$
\int_{z}^{z_{T}} \rho \mathcal{F} d z=\left.\rho \overline{u^{\prime} w^{\prime}}\right|_{z}
$$

where $\mathcal{F}=\rho^{-1} \partial\left(\rho \overline{u^{\prime} w^{\prime}}\right) / \partial z$ and $u^{\prime}$ and $w^{\prime}$ are gravity wave zonal and vertical velocities, thereby enforcing conservation of parameterized momentum flux (Shepherd and Shaw 2004). It is clear from (1) that defining momentum conservation by the amount of gravity wave momentum flux at the model lid (as done here) assumes that all other aspects of the GWD parameterization are conservative. In particular, both the theoretical and numerical formulations must also be conservative and care was taken to ensure this in the model simulations. In the nonconservative configuration the gravity wave momentum flux is assumed to escape to space, and hence the tendency at the highest model level is set equal to zero. This has tended to be the default practice in GCM modeling. Other sources of nonconservation, such as a zonal-mean sponge layer in the presence of GWD, are not considered here.

In what follows, we take a "perfect model" approach: the HIGH_C response is taken as the truth, because its dynamics most closely mimic that of the real atmosphere. Which configuration agrees best with observations is not the point (although as noted above, the HIGH_C configuration is the standard version of the CMAM and has been shown to agree well with observations). For example, if LOW_N was tuned to match observations, then imposing conservation could only make the model depart from observations, but this would not imply that momentum conservation was wrong. In this perfect model approach, differences between the other configurations and HIGH_C are considered as biases. For similar reasons, we only discuss the total GWD response (sum of orographic and nonorographic components) in the two sets of model simulations. Because the two GWD components can be tuned in a variety of ways to obtain the current mean climate (resulting from a lack of constraints from observations), the exact partitioning into orographic and nonorographic contributions is very likely model dependent, and hence the sum is considered to be the most meaningful quantity. We will, however, describe the relative contributions of the two different GWD components in the text when discussing the GWD response.

\section{Impact of momentum conservation on the control climate}

The importance of parameterized gravity waves (both orographic and nonorographic) in determining the structure of the modeled climate is well established. Orographic GWD plays an important role in determining the structure of the zonal-mean zonal wind in the upper troposphere and lower stratosphere, as well as surface pressure, while nonorographic GWD plays a crucial role in the momentum budget of the stratosphere and mesosphere. Not parameterizing these processes leads to intolerable biases in the modeled climate (McFarlane 1987; Garcia and Boville 1994). Here we examine the impact of momentum conservation on the control climate (both the zonal-mean and the surface climate), in particular the indirect effect of dynamical feedbacks resulting from changes in the resolved waves.

We focus first on the Northern Hemisphere, and subsequently on the Southern Hemisphere, in both cases at high latitudes. Figure 1 shows the seasonal cycle of monthly zonal-mean temperature along the 20-hPa surface averaged north of $70^{\circ} \mathrm{N}$ for HIGH_C and HIGH_N (solid lines), and LOW_C (dashed) and LOW_N (dasheddot). The impact of momentum conservation on the seasonal cycle in the high configuration is minimal (difference between solid lines in Fig. 1), and so both HIGH_C and HIGH_N can be regarded as the truth. From here on we will only compare the HIGH_C climate to the two low-lid configurations. When the model lid is lowered to $10 \mathrm{hPa}$ and momentum is conserved, a slight cold bias appears, which is strongest in February (difference between solid and dashed lines in Fig. 1). This cold bias can be considered a direct effect of lowering the lid. When momentum is not conserved in the lowered configuration the cold bias becomes much worse and persists from midfall to midspring (difference between dashed and dash-dot lines in Fig. 1). There is clearly a large impact of momentum conservation on the seasonal cycle of polar cap-averaged temperature at $20 \mathrm{hPa}$ in the lowered configuration.

The zonal-mean structure of the temperature difference between LOW_C and HIGH_C and between LOW_N and HIGH_C in wintertime (DecemberFebruary) is shown, respectively, in Fig. 2, left and right. The slight cold bias in LOW_C relative to HIGH_C, seen in Fig. 1, extends below $20 \mathrm{hPa}$ (though it is weak in amplitude) and from the polar cap into midlatitudes. In contrast, the cold bias in LOW_N extends all the way down to the upper troposphere (to approximately 400 $\mathrm{hPa}$ ) and maximizes over the pole. There is also a significant difference in temperature at the surface near $60^{\circ} \mathrm{N}$. 


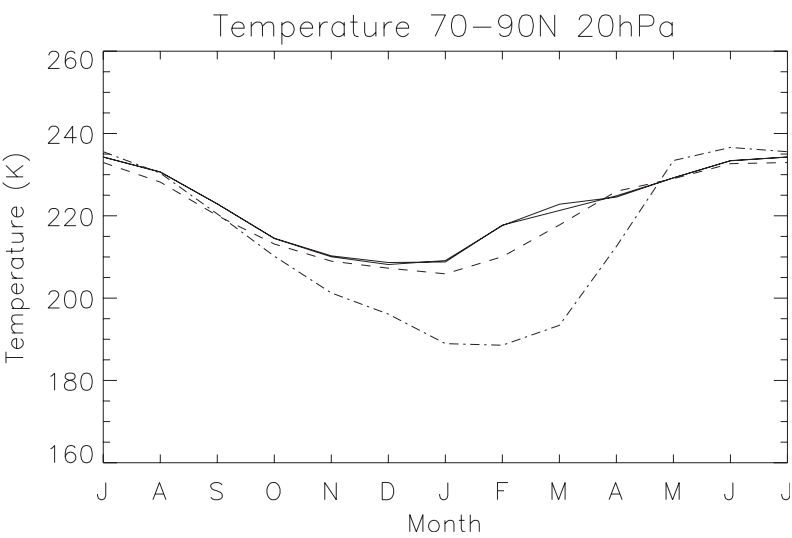

FIG. 1. Seasonal cycle of 40-yr average, monthly mean temperature averaged north of $70^{\circ} \mathrm{N}$ along the $20-\mathrm{hPa}$ surface for HIGH_C and HIGH_N (solid lines), LOW_C (dashed), and LOW_N (dash-dot).

Figure 3 shows the variability of monthly mean temperature at $50 \mathrm{hPa}$ in February and March averaged between $60^{\circ}$ and $90^{\circ} \mathrm{N}$ and its relationship with the meridional heat flux at $100 \mathrm{hPa}$ in January and February averaged between $40^{\circ}$ and $80^{\circ} \mathrm{N}$ (a proxy for the resolved wave forcing affecting the polar stratosphere) for the three configurations. Newman et al. (2001) showed that these two fields are observed to be highly correlated: the implied temperature in the absence of any meridional heat flux (the $y$ intercept of the linear fit) corresponds to radiative equilibrium mitigated by the effects of GWD, and the slope indicates the sensitivity of polar temperature to the resolved wave forcing. The effect of lowering the model lid while conserving momentum on the temperature PDF (shown on the vertical axis) appears to be small, given the variability that is inherent in the 40- member samples. However, when momentum is not conserved the change in the PDF is more dramatic as it becomes positively skewed, with a skewness of $0.8 \pm 0.7$. The relationship in HIGH_C between the temperature and the meridional heat flux is in good agreement with observations (Newman et al. 2001). When the lid is lowered and momentum is conserved, the relationship is mostly preserved. (The linear fits in HIGH_C and LOW_C are in good agreement.) However, when momentum is not conserved the relationship is altered: the $y$ intercept is much colder and the slope is much steeper. The colder intercept is because of a more severe cold bias when westward gravity wave momentum flux is allowed to escape the model lid, and the steeper slope is because of the missing GWD-resolved wave (EPFD) feedbacks discussed further below, which mitigate the effects of the EPFD on polar temperature. Therefore, momentum conservation affects not only the mean climate but also the nature of the interannual variability.

The impact of momentum conservation on the seasonal cycle of polar cap-averaged temperature can be understood in detail by calculating the vertical component of the residual circulation (Andrews et al. 1987) attributable to resolved (planetary) and parameterized (orographic and nonorographic gravity) waves in the high and lowered configurations. The vertical component of the residual circulation is calculated using the downward control principle (Haynes et al. 1991). Figure 4 (top) shows the seasonal cycle of the monthly zonalmean total vertical residual velocity (sum of EPFD and GWD contributions) north of $70^{\circ} \mathrm{N}$ at $20 \mathrm{hPa}$ for HIGH_C (solid), LOW_C (dashed), and LOW_N (dashed-dot). The sum of the two contributions agrees well with the model vertical residual velocity (not shown),
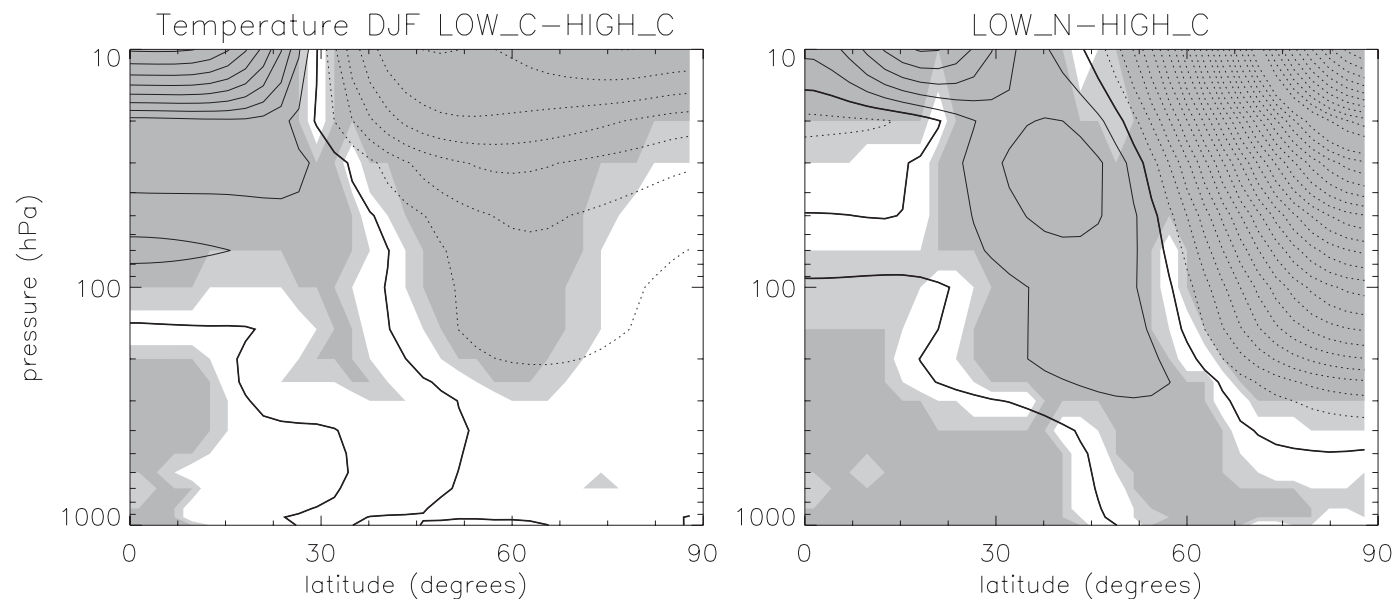

FIG. 2. Zonal-mean temperature difference between (left) LOW_C and HIGH_C and (right) LOW_N and HIGH_C in December-February (40-yr average) in the Northern Hemisphere. Contour interval is $1 \mathrm{~K}$; negative values are dashed. Dark (light) shading denotes confidence at 99\% (95\%) level. 

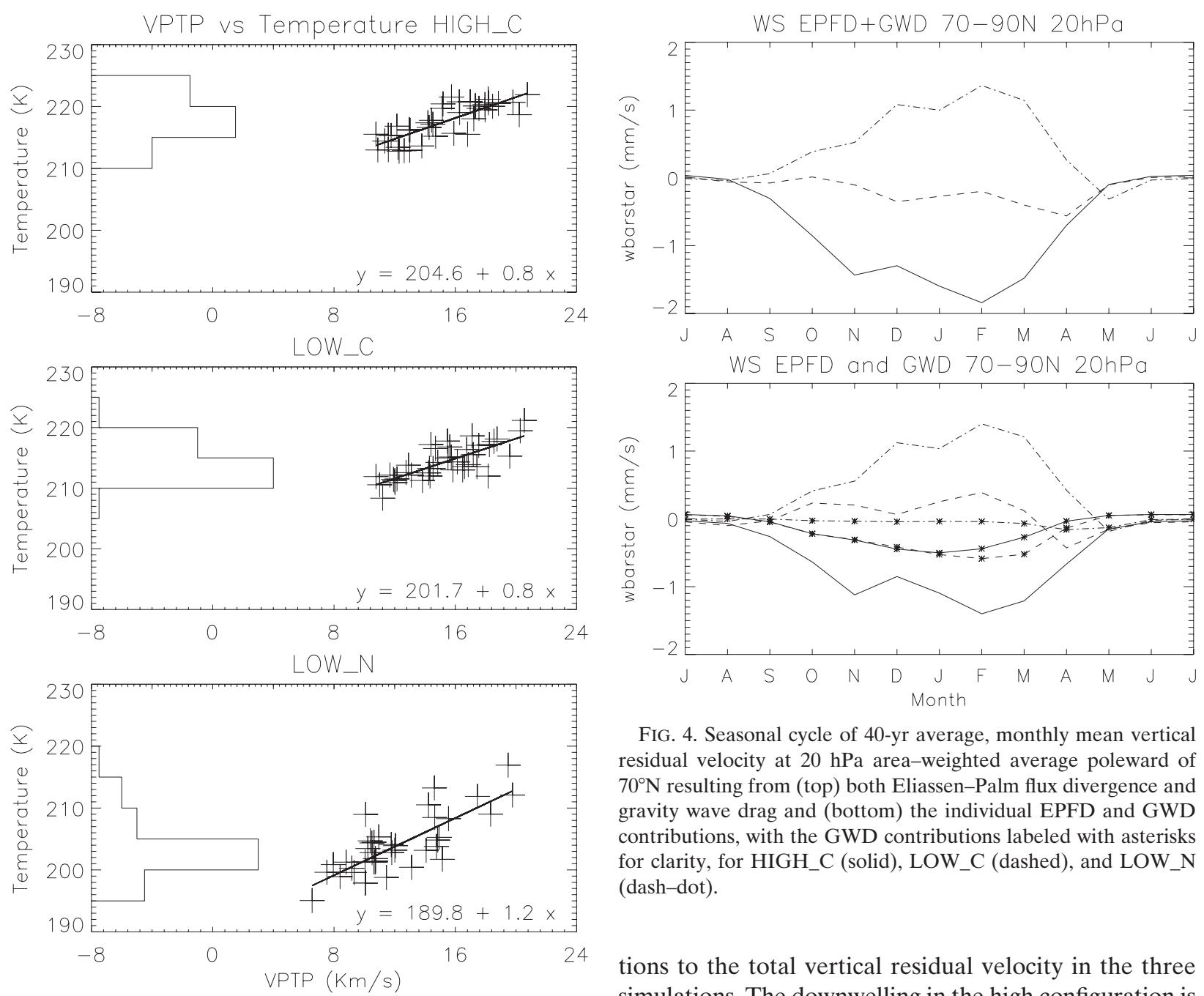

FIG. 3. Meridional heat flux at $100 \mathrm{hPa}$ in January and February averaged between $40^{\circ}$ and $80^{\circ} \mathrm{N}$ versus temperature at $50 \mathrm{hPa}$ in February and March averaged between $60^{\circ}$ and $90^{\circ} \mathrm{N}$ for (top) HIGH_C, (middle) LOW_C, and (bottom) LOW_N. The temperature probability density function $(40 \mathrm{yr})$ is shown on the vertical axis and its linear fit with the meridional heat flux is shown in the bottom-right corner.

confirming that downward control is a reasonable approximation for this diagnostic. The high configuration shows significant downwelling from midfall to midspring (Fig. 4, top, solid line). The effect of lowering the model lid while conserving momentum on the vertical residual circulation is to significantly weaken the polar downwelling (difference between dashed and solid lines in Fig. 4 top). The vertical residual velocity is further distorted when momentum is not conserved; there is a reversal from weak polar downwelling to strong polar upwelling, which persists from midfall to midspring.

To further understand the source of the differences, Fig. 4 (bottom) shows the EPFD and GWD contribu-

FIG. 4. Seasonal cycle of 40-yr average, monthly mean vertical residual velocity at $20 \mathrm{hPa}$ area-weighted average poleward of $70^{\circ} \mathrm{N}$ resulting from (top) both Eliassen-Palm flux divergence and gravity wave drag and (bottom) the individual EPFD and GWD contributions, with the GWD contributions labeled with asterisks for clarity, for HIGH_C (solid), LOW_C (dashed), and LOW_N (dash-dot).

tions to the total vertical residual velocity in the three simulations. The downwelling in the high configuration is mostly EPFD induced with GWD-induced downwelling contributing mostly in the winter season. The GWDinduced downwelling is mostly due to the orographic component. Enforcing conservation of momentum while lowering the model lid ensures that the GWDinduced downwelling is essentially the same as it is in the high configuration. However, the resolved wave contribution is altered when the lid is lowered to $10 \mathrm{hPa}$. In particular, the EPFD-induced downwelling changes to upwelling until early spring when there is downwelling again. It is clear that lowering the model lid has a detrimental effect on the resolved waves. When momentum is not conserved there is virtually no GWD-induced downwelling, which suggests that most of the downwelling at $20 \mathrm{hPa}$ comes from GWD above the model lid (10 hPa), which is not accounted for in LOW_N. The resolved wave contribution is further degraded when momentum is not conserved in the lowered configuration; the EPFDinduced upwelling between midfall and spring becomes larger and persists throughout most of the year. 

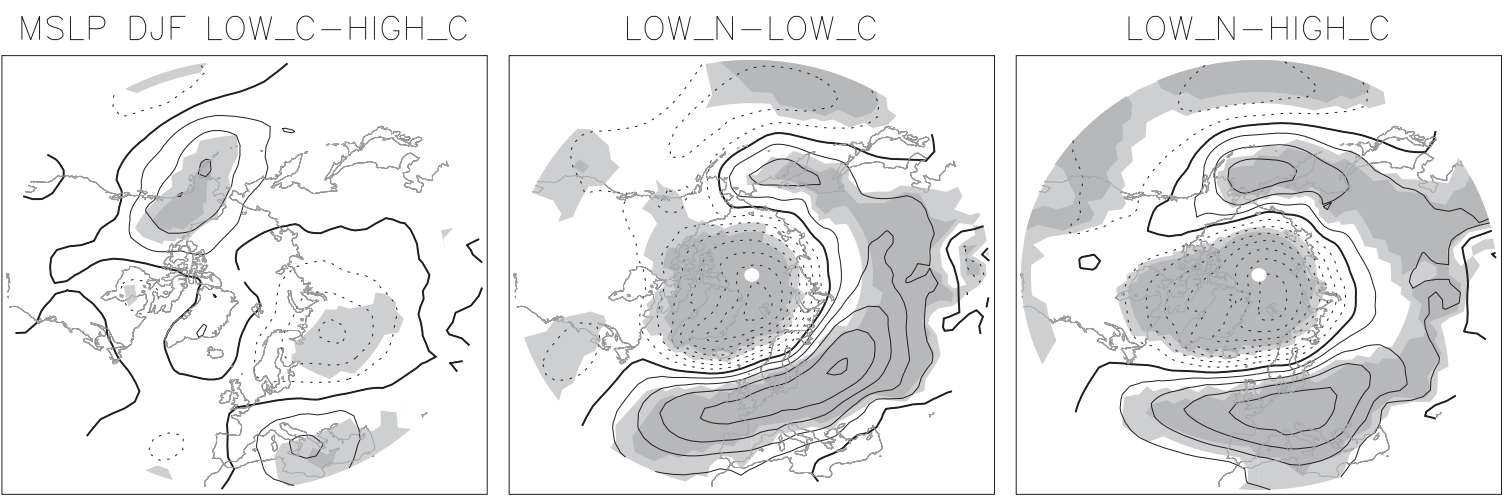

FIG. 5. Difference of mean sea level pressure (40-yr average) in December-February from $30^{\circ}$ to $90^{\circ} \mathrm{N}$ between (left) LOW_C and HIGH_C, (middle) LOW_N and LOW_C, and (right) LOW_N and HIGH_C. Contour interval is $1 \mathrm{hPa}$; negative values are dashed. Dark (light) shading denotes confidence at 99\% (95\%) level.

It is clear that the resolved waves in both lowered configurations are biased relative to HIGH_C. Conserving momentum in the lowered configuration improves the bias relative to LOW_N, but certainly does not eliminate it. The EPFD-induced upwelling in LOW_C and LOW_N is a result of the equatorward and downward deflection of planetary waves at the model lid, an effect that was noted previously by Boville and Cheng (1988, hereafter BC88). There is clearly a large impact of momentum conservation on this bias in the resolved waves; the difference in EPFD-induced upwelling between LOW_N and LOW_C is very large and accounts for most of the large cold bias in Fig. 1. The effect of momentum conservation on the circulation can be summarized as follows: when momentum is conserved in the lowered configuration, the polar night jet is weakened, the spurious equatorward shift of EPFD is reduced, the spurious upwelling is much weaker, and the cold bias at the pole is considerably alleviated.

The impact of momentum conservation in the lowered configuration at the surface is examined in Fig. 5, which shows the difference in mean sea level pressure in wintertime (December-February) between LOW_C and HIGH_C (left), between LOW_N and LOW_C (middle), and between LOW_N and HIGH_C (right). When the model lid is lowered to $10 \mathrm{hPa}$ and momentum is conserved there is very little change in the mean sea level pressure (difference between LOW_C and HIGH_C, see Fig. 5, left). This is consistent with Fig. 2, which showed that in the Northern Hemisphere the effect of lowering the model lid is mostly confined to the region in its vicinity. In contrast, when momentum is not conserved the mean sea level pressure changes significantly (Fig. 5 middle). This extension to the surface was seen in Fig. 2 (right). The difference between LOW_N and LOW_C has an annular structure. The impact of momentum conservation on the surface climate is pre- sumably a result of the dynamical feedbacks (changes in EPFD) on the circulation and accounts for most of the difference between LOW_N and HIGH_C (Fig. 5, right). It is clear that momentum conservation in a 10 -hPa lid model is important for mean sea level pressure as well as stratospheric polar temperatures in the Northern Hemisphere.

Turing now to the Southern Hemisphere, the seasonal cycle of polar cap-averaged temperature is shown in Fig. 6 and is shifted by 6 months relative to Fig. 1. As at the North Pole, there is little impact of momentum conservation on polar cap temperature in the high configuration, and so we do not discuss HIGH_N any further. When the model lid is lowered to $10 \mathrm{hPa}$ and momentum is conserved, while there is little change in the polar cap temperature up to late winter, after that point a cold bias appears which is particularly strong in September-November (difference between solid and dashed lines in Fig. 6). This contrasts with the Northern Hemisphere where the polar cap temperature showed a relatively small impact of lowering the model lid when momentum was conserved (Fig. 1). This suggests that lowering the model lid has a more detrimental effect on temperatures in the Southern versus the Northern Hemisphere. When momentum is not conserved the cold bias worsens (difference between dashed and dashdot lines in Fig. 6) and persists from winter to spring. However, the difference between LOW_N and LOW_C is less than it was at the North Pole.

The zonal-mean structure of the temperature difference between LOW_C and HIGH_C and LOW_N and HIGH_C in wintertime (June-August) in the Southern Hemisphere is shown in Fig. 7. As at the North Pole, the cold bias at $20 \mathrm{hPa}$ in LOW_C, relative to HIGH_C, extends below $20 \mathrm{hPa}$ and into midlatitudes. Unlike at the North Pole, there is an extension of the bias toward the surface around $60^{\circ} \mathrm{S}$. The cold bias in LOW_N also 


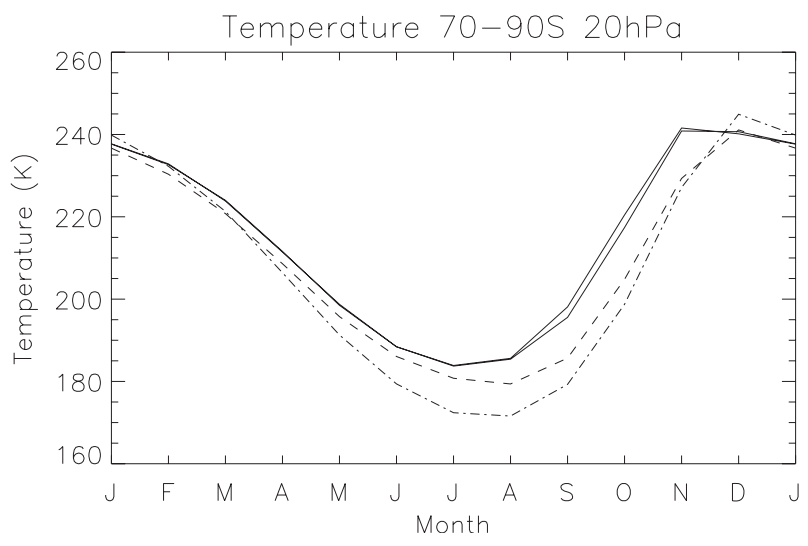

FIG. 6. As in Fig. 1, but south of $70^{\circ}$ S. Note the months are shifted by 6 months relative to Fig. 1 .

extends below $20 \mathrm{hPa}$ and it maximizes at the pole. The bias in LOW_N is slightly weaker, both at the pole and at the surface, than it was at the North Pole.

Figure 8 shows the variability of monthly mean temperature at $50 \mathrm{hPa}$ in August and September averaged between $60^{\circ}$ and $90^{\circ} \mathrm{S}$ and its relationship with the meridional heat flux at $100 \mathrm{hPa}$ in July and August averaged between $40^{\circ}$ and $80^{\circ} \mathrm{S}$ for the three configurations. In the Southern Hemisphere, the PDFs of polar temperatures at $50 \mathrm{hPa}$ for both LOW_C and LOW_N are not inconsistent with HIGH_C, given the sample sizes. [The positive skew of the LOW_N PDF found in the Northern Hemisphere is however found at higher levels in the Southern Hemisphere (e.g., at $20 \mathrm{hPa}$ ).] When the lid is lowered and momentum is conserved, the relationship between the temperature and the meridional heat flux is not as well preserved as in the Northern Hemisphere. The $y$ intercepts are in good agreement; however, the slopes are very different. The change in slope suggests that the interaction between the mean flow (the polar

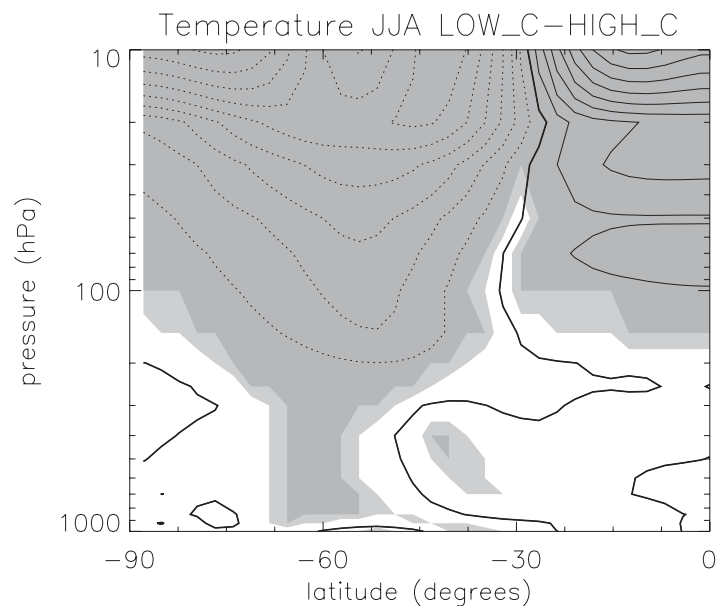

vortex) and the resolved waves (EPFD) is altered by the low lid. This is discussed further below. When momentum is not conserved the $y$ intercept becomes colder and the magnitude of the slope increases, as in the Northern Hemisphere. Thus, in the Southern Hemisphere, the error from nonconservation acts oppositely to that from the low lid, to the extent that, for this diagnostic, LOW_N actually agrees better with HIGH_C than LOW_C does. However, this is only because of compensating errors.

The worse agreement of polar cap-averaged temperatures between LOW_C and HIGH_C in the Southern Hemisphere compared to the Northern Hemisphere can be understood by looking at the vertical residual velocity over the South Pole. Figure 9 shows the downward control-derived total vertical residual velocity (top) and EPFD and GWD contributions to the vertical residual velocity (bottom), as in Fig. 4, but for the Southern Hemisphere and shifted by 6 months. In the high configuration, there is downwelling at $20 \mathrm{hPa}$ throughout the year. At the South Pole the nonorographic contribution to the GWD-induced downwelling is larger than at the North Pole, and represents approximately onequarter of the total downwelling. As was the case at the North Pole, enforcing momentum conservation when lowering the model lid ensures that the GWD-induced downwelling is not significantly altered. However, when momentum is not conserved this downwelling is not accounted for (there is virtually no GWD-induced downwelling in LOW_N). Figure 9 (bottom) shows that there is a large impact of lowering the model lid to $10 \mathrm{hPa}$ while conserving momentum on the EPFD contribution in the Southern Hemisphere. In particular, the polar downwelling changes to weak polar upwelling from midfall to late spring, and the maximum downwelling is weakened and shifted from November to December. The EPFD-induced upwelling and the shift in the timing

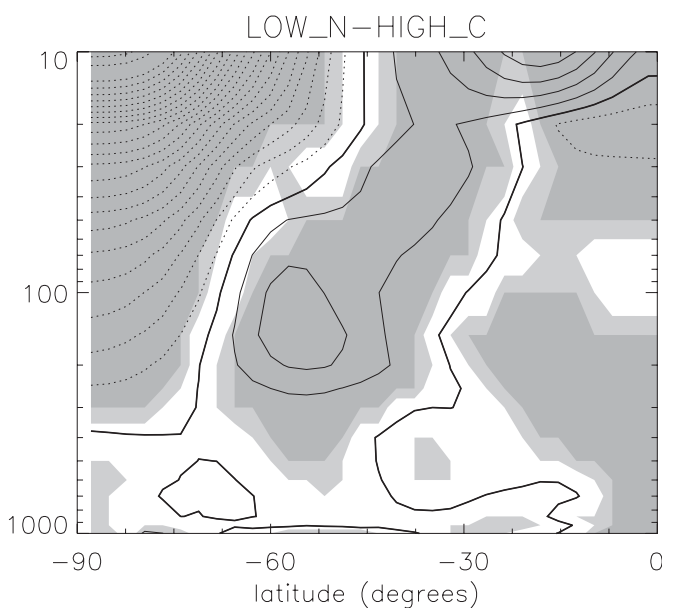

FIG. 7. As in Fig. 2, but for June-August in the Southern Hemisphere. 

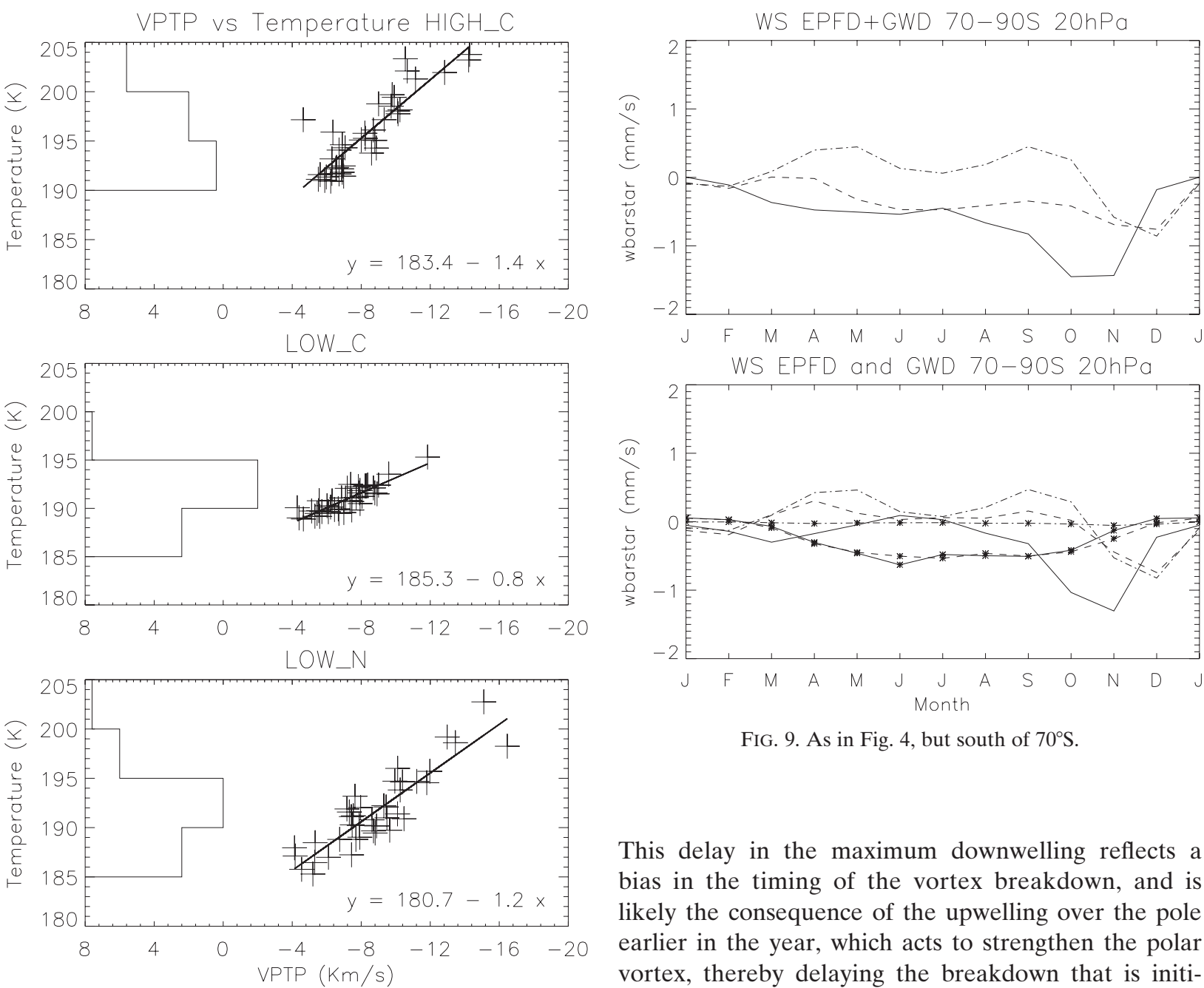

FIG. 8. Meridional heat flux at $100 \mathrm{hPa}$ in July and August averaged between $40^{\circ}$ and $80^{\circ} \mathrm{S}$ versus temperature at $50 \mathrm{hPa}$ in August and September averaged between $60^{\circ}$ and $90^{\circ} \mathrm{S}$ for (top) HIGH_C, (middle) LOW_C, and (bottom) LOW_N. The temperature probability density function $(40 \mathrm{yr}$ ) is shown on the vertical axis and its linear fit with the meridional heat flux is shown in the bottom right-hand corner.

of the maximum downwelling are both the cause of the cold pole bias in late winter (relative to HIGH_C) and the reason it continues into September-November (Fig. $6)$. When momentum is not conserved the bias in the EPFD contribution worsens; there is even more EPFDinduced upwelling though there is no change in the timing of the maximum downwelling (difference between dashed and dash-dot EPFD lines in Fig. 9). It is clear that the impact of momentum conservation on the EPFD contribution is to alleviate some of the bias in the resolved waves, though the bias in the timing of the maximum downwelling is not alleviated and the impact is less than it was in the Northern Hemisphere.

This delay in the maximum downwelling reflects a bias in the timing of the vortex breakdown, and is likely the consequence of the upwelling over the pole earlier in the year, which acts to strengthen the polar vortex, thereby delaying the breakdown that is initiated by radiative damping (instead of EPFD-induced downwelling).

Figure 10 shows the corresponding Southern Hemisphere wintertime (June-August) difference of mean sea level pressure between LOW_C and HIGH_C (left), LOW_N and LOW_C (middle), and LOW_N and HIGH_C (right). Unlike in the Northern Hemisphere, when the model lid is lowered and momentum is conserved (Fig. 10, left) there is a larger region of significant change in mean sea level pressure. This impact on the surface can be seen in Fig. 7 (left). In contrast, in the Northern Hemisphere the impact of lowering the lid was smaller and confined to the region near the lid. When momentum is not conserved the mean sea level pressure is distorted further (Fig. 10, middle). The combined effect of nonconservation of momentum and the lowered lid (Fig. 10, right) is not as strong as in the Northern Hemisphere because the two errors tend to offset each other. Thus, while momentum conservation in a $10-\mathrm{hPa}$ lid model is important for mean sea level pressure, in the Southern Hemisphere it cannot 

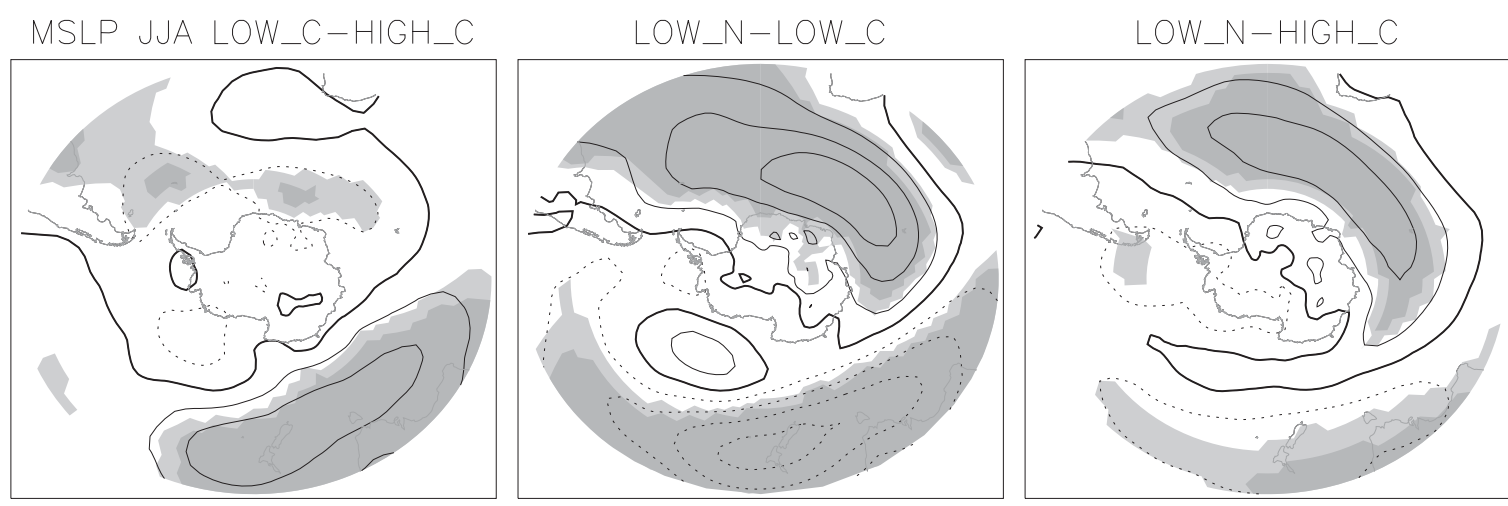

FIG. 10. As in Fig. 5 but for June-August and $30^{\circ}$ to $90^{\circ} \mathrm{S}$.

alleviate the bias associated with the effect of the low lid on the resolved waves.

This discrepancy in the ability of LOW_C to capture the control climate in HIGH_C in the two hemispheres during winter and spring (Figs. 1-5 versus Figs. 6-10), which is associated with differences in the biases in the resolved waves, is presumably attributable to the difference in the strength of the polar vortex between the two hemispheres. Because planetary wave breaking occurs at lower altitudes in the Northern Hemisphere, it is easier for LOW_C to capture more of the planetary wave-induced downwelling that occurs in HIGH_C through a correction of the bias in the polar vortex by the GWD-induced downwelling. However, in the Southern Hemisphere, where the jet is stronger and planetary wave dissipation occurs higher in the atmosphere, the bias is more difficult to correct.

The severe cold bias in LOW_N in the Northern Hemisphere in winter is comparable in magnitude to the $30-\mathrm{K}$ cold pole bias reported by BC88 in their 2-yr perpetual January model-lid height sensitivity simulations. BC88 used two lid height configurations: 10 and $0.5 \mathrm{hPa}$. An important difference between the simulations performed by $\mathrm{BC} 88$ and the results shown here is that the model used by BC88 did not include any parameterized GWD. Thus, there was no parameterized momentum flux to conserve. To alleviate the severe cold pole bias in their lowered $(10 \mathrm{hPa})$ configuration associated with planetary wave deflection at the lid, BC88 applied a Rayleigh drag sponge layer in the up- permost levels (100-10 hPa). Even with the Rayleigh drag sponge layer the cold bias was $20 \mathrm{~K}$ at $20 \mathrm{hPa}$. BC 88 discussed the importance of a source of drag in their lowered configuration to help correct the behavior of the resolved waves near the model lid. However they noted that it is very difficult to damp planetary waves because of their long vertical wavelengths, and thus all low-lid configurations would suffer from similar biases in the planetary wave-induced residual vertical velocity. This is what we see here even in the conservative case.

Note that even though Rayleigh drag helps to alleviate the biases in the zonal-mean climate, it is a very crude representation of GWD and does not conserve momentum. Furthermore, the response of Rayleigh drag to an imposed perturbation is inherently unphysical (Shepherd et al. 1996; Shepherd and Shaw 2004). Thus, conserving parameterized gravity wave momentum flux is clearly the most physically correct way to apply GWD in a low-lid model.

\section{Impact of momentum conservation on the response to idealized ozone depletion}

To explore the impact of momentum conservation on the modeled response to a climate perturbation, the seasonal cycle of prescribed ozone in the model simulations presented in section 3 was altered to include idealized ozone depletion in the Southern Hemisphere. The ozone depletion was prescribed as a fractional loss with the following analytical structure:

$$
\alpha(p)=\left\{\begin{aligned}
\cos ^{2}\left(\frac{\pi}{2} \frac{(30 \mathrm{hPa}-p)}{(30 \mathrm{hPa}-60 \mathrm{hPa})}\right) & 60 \mathrm{hPa} \geq p \geq 30 \mathrm{hPa} \\
\cos ^{2}\left(\frac{\pi}{2} \frac{(1.0}{(120 \mathrm{hPa}-300 \mathrm{hPa})}\right) & 120 \mathrm{hPa} \geq p \geq 60 \mathrm{hPa}, \\
300 \mathrm{hPa} & \geq p \geq 120 \mathrm{hPa}
\end{aligned}\right.
$$




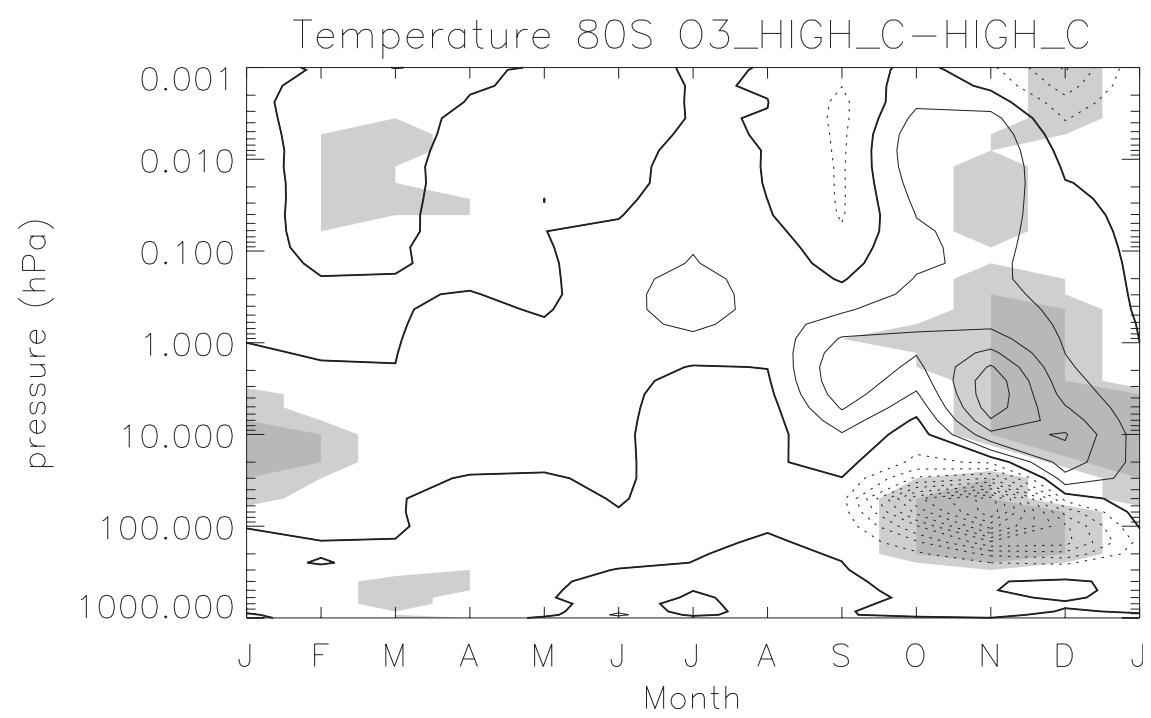

FIG. 11. Zonal and monthly mean temperature response at $80^{\circ} \mathrm{S}$ to the imposed ozone depletion in HIGH_C. Contour interval is $1 \mathrm{~K}$; negative values are dashed. Dark (light) shading denotes confidence at $99 \%(95 \%)$ level.

$$
\beta(\phi)=\left\{\begin{array}{cc}
1.0 & \phi \geq 70^{\circ} \mathrm{S} \\
\cos ^{2}\left(\frac{\pi}{2} \frac{\left(\phi-70^{\circ} \mathrm{S}\right)}{\left(65^{\circ} \mathrm{S}-70^{\circ} \mathrm{S}\right)}\right) & 70^{\circ} \mathrm{S} \geq \phi \geq 65^{\circ} \mathrm{S},
\end{array}\right.
$$

$$
\gamma\left(t_{d}\right)=\left\{\begin{array}{rlrl}
\cos ^{2}\left(\frac{\pi}{2} \frac{\left(t_{d}-274\right)}{30}\right) & 273 & \geq t_{d} \geq 244 \\
\cos ^{2}\left(\frac{\pi}{2} \frac{\left(t_{d}-304\right)}{30}\right) & 304 & \geq t_{d} \geq 274 \\
334 & \geq t_{d} \geq 305 .
\end{array}\right.
$$

Here $p$ is pressure, $\phi$ is latitude, and $t_{d}$ is day of the year.

The temperature response to ozone depletion is well documented by radiosondes and satellites (Randel and $\mathrm{Wu}$ 1999). The observed radiative cooling resulting from ozone depletion is accompanied and followed by a warming at higher levels, which is understood to be a dynamical response to the cooling (Mahlman et al. 1994). In particular, the warming has been attributed to increased downwelling by planetary and gravity waves in response to the zonal-wind changes induced by the radiative cooling (Manzini et al. 2003). For the planetary waves, a delay in vortex breakdown allows planetary waves to propagate into the stratosphere later in the season. For the gravity waves, the increase in downwelling is a generic result of wave filtering by an imposed zonal wind perturbation (Holton 1983): there is an increase in the amount of westward drag at upper levels (resulting from a stronger eastward wind maximum below) and an increase in eastward drag at lower levels, which results in downwelling (adiabatic warming) over the pole. The surface response to ozone depletion is also well documented. The depletion induces a cooling trend over the Antarctic interior and a warming trend over the peninsula, consistent with a positive southern annular mode response (Thompson and Solomon 2002). The impact of momentum conservation and model-lid height on these responses to ozone depletion is the issue addressed here.

As discussed in the introduction, an analogous ozone depletion perturbation experiment was performed by SS07 using a zonally symmetric model and focusing solely on the nonorographic GWD response. Here we consider the extension of the lid height aspect of those results to three dimensions with a fully variable basic state, including planetary waves and parameterized orographic GWD.

The temperature response at $80^{\circ} \mathrm{S}$ associated with the imposed ozone depletion in the high configuration (difference between O3_HIGH_C and HIGH_C) is shown in Fig. 11. The magnitude of the maximum negative temperature response is comparable to observations (Randel and Wu 1999). The effects of a lowered lid and nonconservation of momentum on this temperature response (below $10 \mathrm{hPa}$ ) are shown in Fig. 12 (middle and bottom, respectively). (The high configuration response below $10 \mathrm{hPa}$ is plotted in the top panel of Fig. 12 for reference.) When the model lid is lowered and momentum is conserved (middle panel), the maximum negative temperature response is strengthened and persists from November through to the end of January. This response is degraded further when momentum is not conserved. In particular, the maximum negative temperature is even stronger, has a deeper vertical extent, and is even more persistent. 


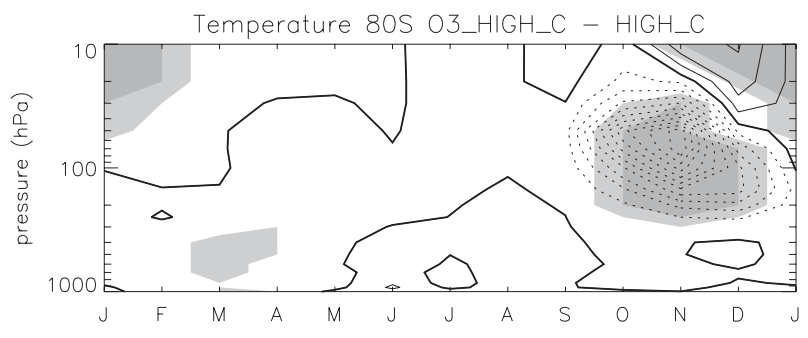

O3_LOW_C - LOW_C

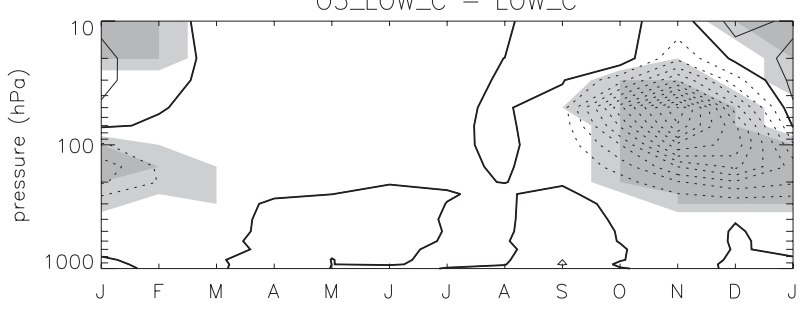

O3_LOW_N - LOW_N

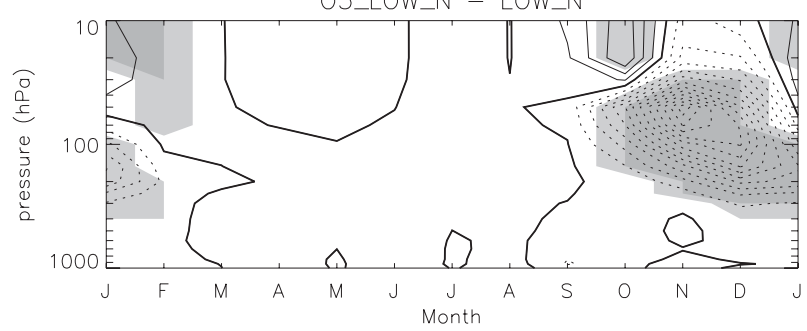

FIG. 12. (top) Zonal and monthly mean temperature response at $80^{\circ} \mathrm{S}$ below $10 \mathrm{hPa}$ to the imposed ozone depletion as in Fig. 11, and the differences between the response in Fig. 11 and those in (middle) LOW_C and (bottom) LOW_N. Contour interval is $1 \mathrm{~K}$; negative values are dashed. Dark (light) shading denotes at $99 \%$ (95\%) level.

The zonal wind response to the imposed ozone depletion averaged over December and January is shown in Fig. 13 for HIGH_C below $10 \mathrm{hPa}$ (top), LOW_C (middle), and LOW_N (bottom). The response in the high configuration shows a significant eastward perturbation that extends to the surface. Lowering the model lid while conserving momentum leads to a strengthened eastward response (difference between Fig. 13, top and middle), which is consistent with the stronger temperature response above $100 \mathrm{hPa}$ (Fig. 12, middle); however, the response below $100 \mathrm{hPa}$ is unaltered. When momentum is not conserved the eastward zonal wind response is further degraded; the response above 100 $\mathrm{hPa}$ is strengthened and its extension to the surface becomes less significant (difference between Fig. 13, middle and bottom).

The surface response to ozone depletion as measured by the difference in mean sea level pressure averaged over December and January is shown in Fig. 14 for HIGH_C (left), LOW_C (middle), and LOW_N (right). The surface response to ozone depletion in the high configuration is in good agreement with observations
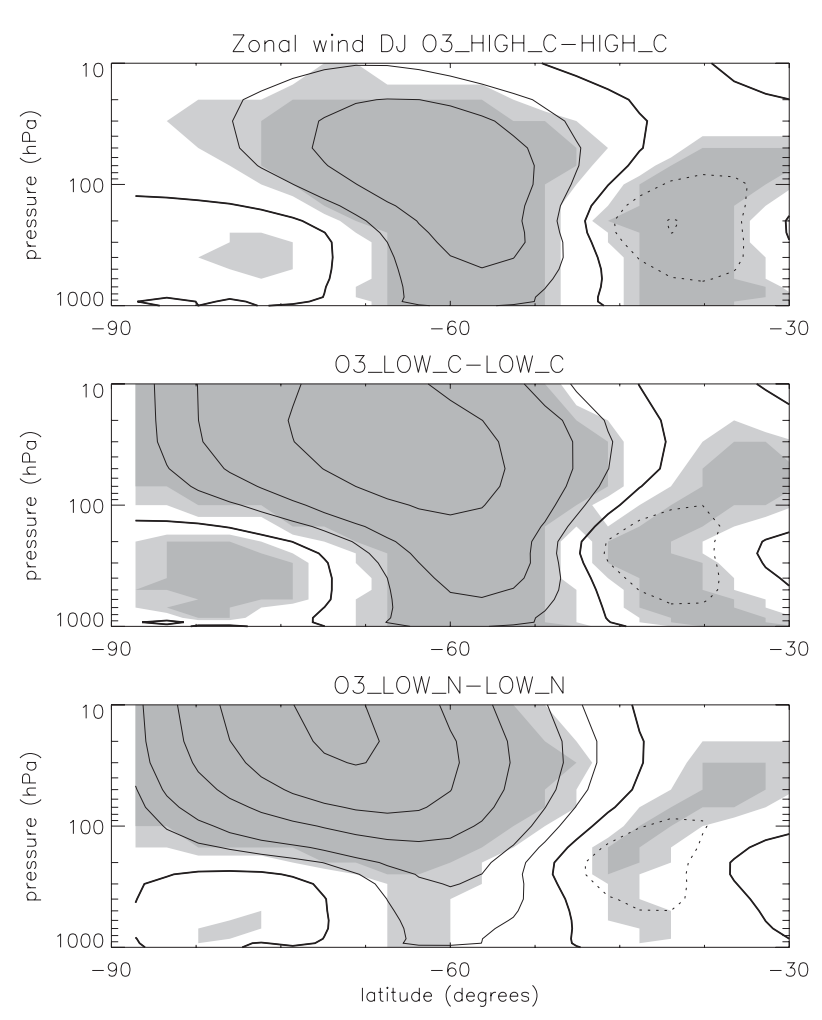

FIG. 13. Zonal and monthly mean zonal wind response in December and January to the imposed ozone depletion in (top) HIGH_C, (middle) LOW_C, and (bottom) LOW_N. Contours: $\pm 1, \pm 2, \pm 4, \pm 6$, and $\pm 8 \mathrm{~m} \mathrm{~s}^{-1}$; negative values are dashed. Dark (light) shading denotes confidence at 99\% (95\%) level.

(Thompson and Solomon 2002). When the lid is lowered to $10 \mathrm{hPa}$ and momentum is conserved the negative response over the polar cap becomes slightly larger and a large positive response appears between $30^{\circ}$ and $60^{\circ} \mathrm{S}$ (Fig. 14, middle). This large positive response is considered as a direct effect of lowering the lid and is similar to the difference seen in the control climate sea level pressure between LOW_C and HIGH_C (Fig. 10, left). When momentum is not conserved, the surface response is weaker and less annular (Fig. 14, right versus middle). Thus, momentum conservation is important for capturing the surface response to ozone depletion in the lowered configuration; however, there still appears to be biases associated with the effect of the low model lid on the resolved waves (in the control climate) as discussed in the previous section.

The impact of momentum conservation on the dynamical response in December and January to ozone depletion can be further understood by investigating the response of the vertical residual velocity in the different configurations. The dynamical warming observed to lag the ozone-induced cooling is well modeled by HIGH_C 

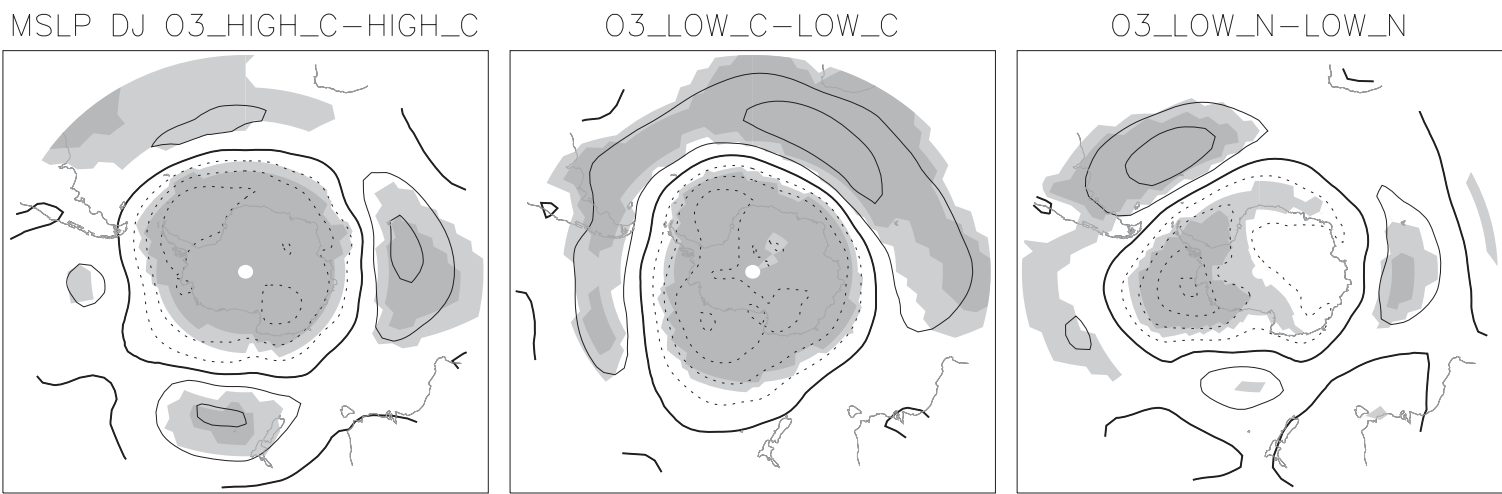

FIG. 14. Response of mean sea level pressure from $30^{\circ}$ to $90^{\circ} \mathrm{S}$ to the imposed ozone depletion in (left) HIGH_C, (middle) LOW_C, and (right) LOW_N. Contour interval is $1 \mathrm{hPa}$; negative values are dashed. Dark (light) shading denotes confidence at $99 \%(95 \%)$ level.

(Fig. 11), and extends above $1 \mathrm{hPa}$. In agreement with Manzini et al. (2003), we find this to be a dynamical response to the imposed ozone depletion. Figure 15 shows the vertical residual velocity response to ozone depletion south of $70^{\circ} \mathrm{S}$ from August to January for the high configuration. There is a clear increase in downwelling in November and December, as argued above.

The dynamical response to the imposed ozone depletion for all configurations is shown in Fig. 16. (The high configuration response below $10 \mathrm{hPa}$ is plotted in the top panel of Fig. 16 for reference.) When the lid is lowered to $10 \mathrm{hPa}$ and momentum is conserved the maximum downwelling is shifted from November to December (Fig. 16, middle). However, the magnitude of the response is not significantly altered. The bias in the timing of the maximum downwelling response reflects the bias in the EPFD-induced downwelling in the control climate discussed in the previous section. When momentum is not conserved the downwelling response maximizes in October and there is no statistically significant response from November to January (Fig. 16, bottom).

To further understand the source of the differences in the dynamical responses to ozone depletion, the downwelling response is partitioned into the EPFD and the GWD contributions using the downward control principle, as was done for the control climate (Figs. 4 and 9). The response in EPFD-induced downwelling from August to January is shown in the left-hand column of Fig. 17 for HIGH_C (top), LOW_C (middle), and LOW_N (bottom). In the high configuration it is

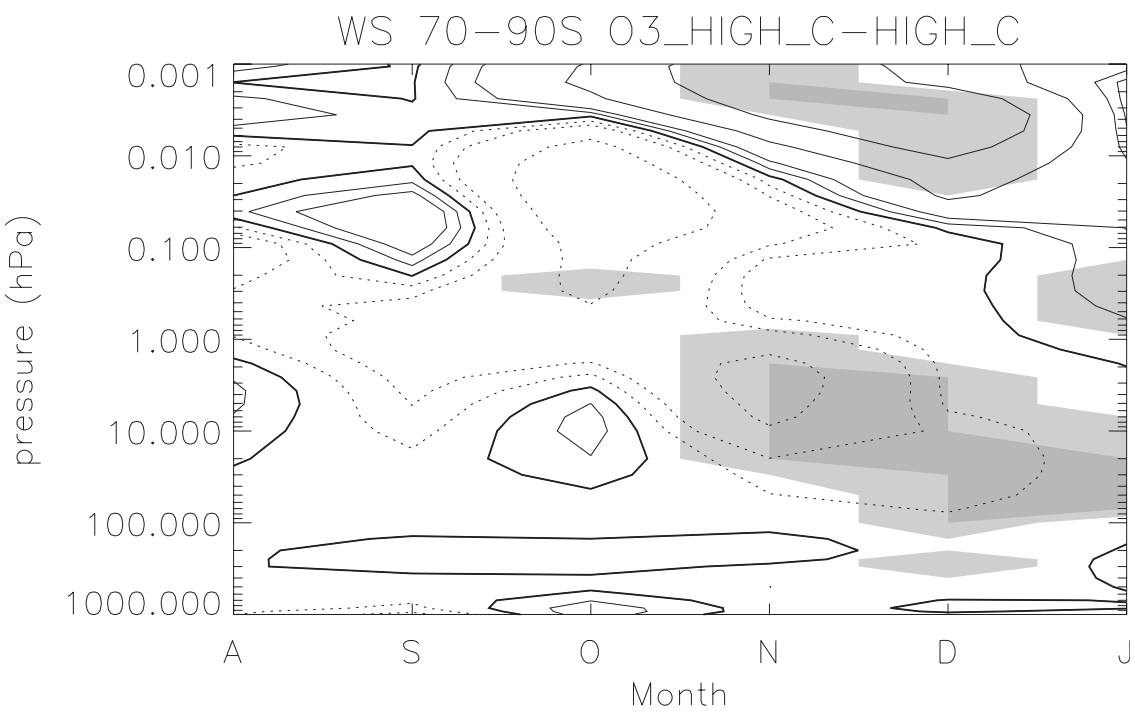

FIG. 15. Response of monthly mean residual vertical velocity to the imposed ozone depletion, area weighted poleward of $70^{\circ} \mathrm{S}$ in $\mathrm{HIGH}_{-}$. Contours: $\pm 0.1, \pm 0.2, \pm 0.5, \pm 1.0$, and $\pm 2.0 \mathrm{~mm} \mathrm{~s}^{-1}$; negative values are dashed. Dark (light) shading denotes confidence at $99 \%$ $(95 \%)$ level. 

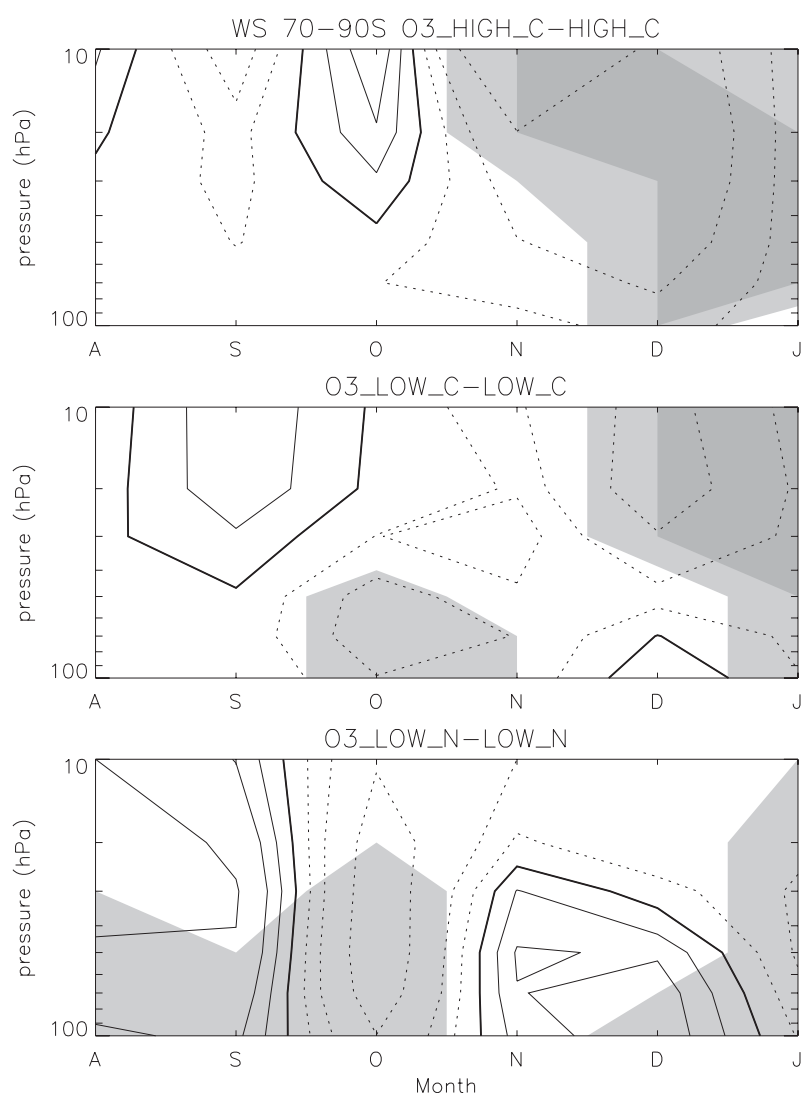

FIG. 16. Response of monthly mean residual vertical velocity to the imposed ozone depletion from 100 to $10 \mathrm{hPa}$, area weighted poleward of $70^{\circ} \mathrm{S}$ in (top) $\mathrm{HIGH}$ _C, (middle) LOW_C, and (bottom) LOW_N. Contours: $\pm 0.05, \pm 0.1, \pm 0.2$, and $\pm 0.5 \mathrm{~mm} \mathrm{~s}^{-1}$; negative values are dashed. Dark (light) shading denotes confidence at $99 \%(95 \%)$ level.

clear that a large contribution to the total downwelling shown in Fig. 16 (top) comes from EPFD. When the model lid is lowered and momentum is conserved, the EPFD-induced downwelling response is shifted by half a month and is weakened below $70 \mathrm{hPa}$. A lagged downwelling response was also seen in the control climate and was attributable to biases in the resolved waves resulting from the low model lid. When momentum is not conserved, the downwelling in November and December is not statistically significant and does not extend below $40 \mathrm{hPa}$ (Fig. 17, bottom left). It is clear that momentum conservation partially corrects the bias in the resolved wave response to ozone depletion, in part by partially correcting the bias in the control climate.

The GWD contribution to the residual vertical velocity from August to January is shown in the right-hand column of Fig. 17. The high configuration response to ozone depletion shows strong downwelling, in agreement with the physically correct response of GWD to an imposed polar radiative cooling identified by SS07. The
GWD-induced downwelling response has roughly equal contributions from the orographic and nonorographic components. When the model lid is lowered and momentum is conserved, the GWD-induced downwelling response is slightly prolonged (Fig. 17, middle right). However, when momentum is not conserved, there is virtually no GWD-induced downwelling in response to ozone depletion (Fig. 17, bottom right), and thus the response is completely altered. This impact of momentum conservation on the GWD response is consistent with the results of SS07 (see their Fig. 12). SS07 found that when momentum was not conserved in a low-lid model the GWD response to an imposed polar cooling was weak upwelling instead of downwelling over the pole. This upwelling was a consequence of allowing gravity wave momentum flux to escape to space: more westward momentum flux escapes in the ozone holeperturbed climate than in the unperturbed climate, leading to a deficit of westward drag, and hence less downwelling.

\section{Summary and discussion}

Every implementation of a flux-based GWD parameterization requires that a decision be made regarding what to do with the gravity wave momentum flux at the model lid. The usual practice is to let the flux escape rather than be conserved. Yet the implications of this decision seem never to have been investigated with a comprehensive GCM. Here we have used the CMAM to quantify the impact of momentum conservation on modeled climate and on its response to idealized ozone depletion. Two model-lid height configurations have been used: the standard high-lid configuration with the model lid at $0.001 \mathrm{hPa}$, and a configuration with the lid at $10 \mathrm{hPa}$. In all cases, conservation of momentum is enforced by depositing the parameterized gravity wave momentum flux at the model lid in the uppermost model level, within each model grid box. The different model configurations allow for a clear separation of the impact of momentum conservation from the effects of lowering the model lid.

We find very little impact of momentum conservation on the control climate and its variability in the high configuration. This insensitivity is expected given that there is very little gravity wave momentum flux left at $0.001 \mathrm{hPa}$. However, the impact is considerable in the lowered configuration, and overall we find that momentum conservation brings the simulated climate in closer agreement with the climate in the high-lid configuration, regarded here as the truth. When the model lid is lowered from 0.001 to $10 \mathrm{hPa}$, the control climate and its variability are not significantly altered in the Northern Hemisphere, provided momentum is 

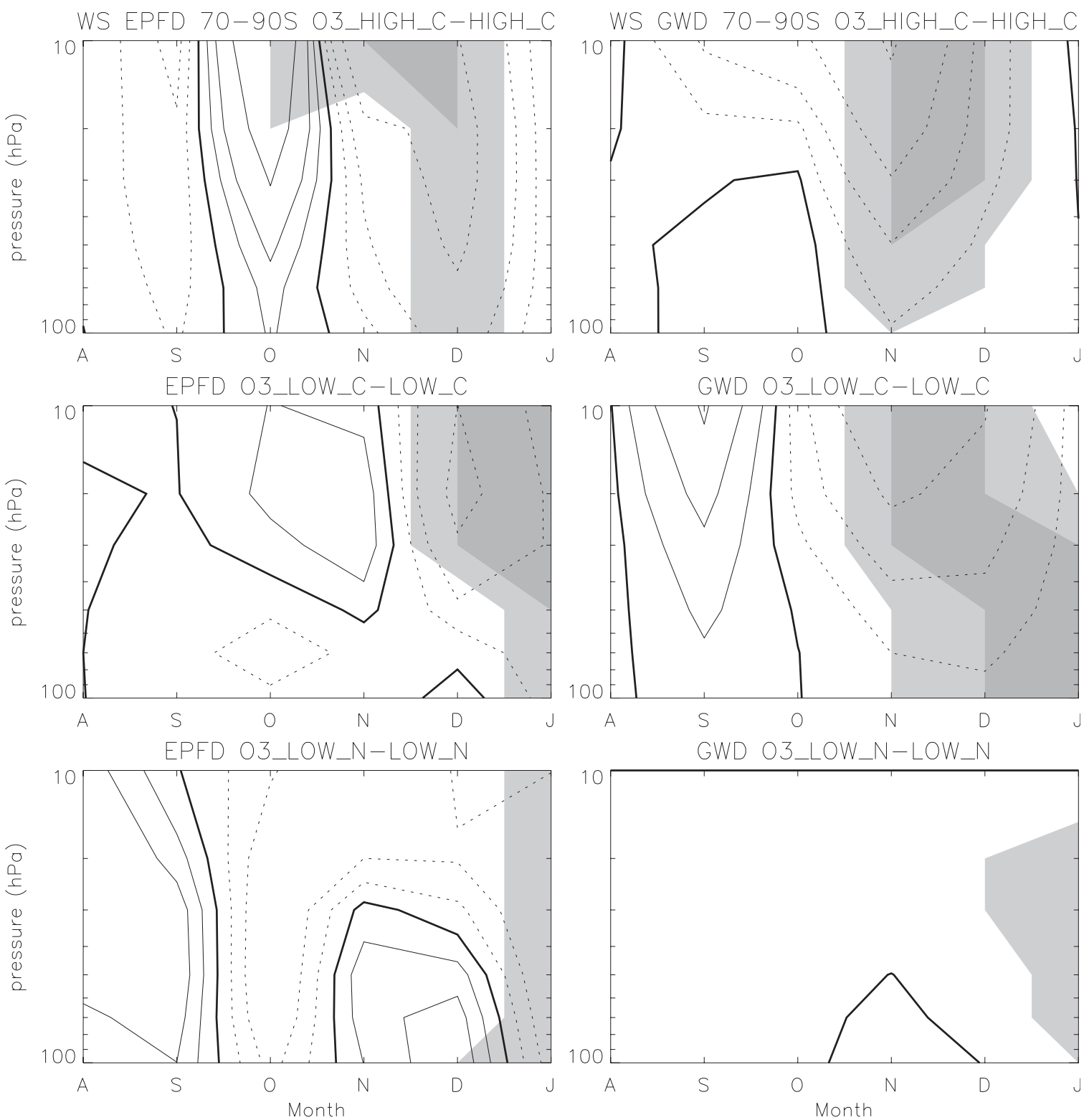

FIG. 17. Response of monthly mean residual vertical velocity to the imposed ozone depletion, area weighted poleward of $70^{\circ} \mathrm{S}$, attributable to (left) planetary waves and (right) gravity waves for (top) HIGH_C, (middle) LOW_C, and (bottom) LOW_N. Contours: $\pm 0.02, \pm 0.05, \pm 0.1, \pm 0.2$, and $\pm 0.5 \mathrm{~mm} \mathrm{~s}^{-1}$; negative values are dashed. Dark (light) shading denotes confidence at $99 \%(95 \%)$ level.

conserved. In the Southern Hemisphere, however, lowering the model lid has a larger impact on polar cap temperatures even when momentum is conserved. The climate develops a cold pole bias in SeptemberNovember resulting from biases in the resolved waves (as discussed further below). While the distribution of polar cap-averaged temperature at $50 \mathrm{hPa}$ does not appear to be significantly altered at the South Pole (within the limits of the sample sizes), the relationship between 50-hPa polar temperature and the midlatitude meridi- onal heat flux at $100 \mathrm{hPa}$ is altered. This suggests that in the Southern Hemisphere, the interaction between the mean flow and the resolved waves (EPFD) is altered by the low lid. When momentum is not conserved, the seasonal cycle of polar temperatures is significantly degraded in both hemispheres. In particular, a very large cold pole bias occurs at both poles, which extends well into the upper troposphere. This is partly the result of missing GWD-induced downwelling (because of the gravity wave momentum flux allowed to escape the 
model domain), and partly the resulting feedback on the resolved EPFD. There is a large impact of momentum conservation on the variability of polar temperature in the Northern Hemisphere: when momentum is not conserved the PDF becomes positively skewed. In both hemispheres, the relationship between polar temperature and meridional heat flux is significantly altered by the loss of GWD associated with nonconservation, in similar ways.

The effect of momentum conservation in the lowered configuration extends all the way to the surface. Over the northern extratropics in wintertime, lowering the model lid does not lead to significant changes in mean sea level pressure when momentum is conserved. However, in the Southern Hemisphere there is a larger impact at the surface, particularly at midlatitudes. When momentum is not conserved the mean sea level pressure is significantly degraded in both hemispheres (it is degraded even further in the Southern Hemisphere). In the Northern Hemisphere, nonconservation of momentum results in a significant annular response. It is clear that in a $10-\mathrm{hPa}$ lid model momentum conservation is important for mean sea level pressure, although even with momentum conservation biases in the resolved waves affect the surface response in the Southern Hemisphere.

As noted by BC88, all models with a lid in the midstratosphere suffer from the deflection of resolved waves at the model lid and thus a reduction in the amount of downwelling over the pole as compared to high-lid models. This effect of lowering the model lid on the resolved waves is seen here in both hemispheres. When the lid is lowered with momentum conservation the EPFD-induced vertical residual velocity changes from downwelling to weak upwelling in the Northern Hemisphere. In the Southern Hemisphere the downwelling in fall and early spring also changes to weak upwelling. There is also a weakening of the downwelling in late spring and a shift in the maximum downwelling from November to December, which is related to the cold bias in the spring. The deflection of resolved waves is exacerbated when momentum is not conserved and this leads to a strong polar upwelling in both hemispheres.

Enforcing conservation of momentum ensures that the GWD-induced downwelling is not altered when the lid is lowered to $10 \mathrm{hPa}$. This downwelling, attributable mostly to orographic GWD in the Northern Hemisphere and to a combination of orographic and nonorographic in the Southern Hemisphere, acts to weaken the vortex in the lowered configuration and corrects some of the errors in planetary wave propagation near the model lid. This indirect feedback of momentum conservation on the resolved waves via the mean flow is larger in the Northern as compared with the Southern
Hemisphere. When momentum is not conserved there is no GWD-induced downwelling to keep the polar vortex weak enough to admit planetary wave propagation, and the deflection of resolved waves is exacerbated.

The high configuration response to the idealized ozone depletion includes a cooling between 200 and $20 \mathrm{hPa}$ from October to December, which agrees well with observations, and a robust dynamical warming between 40 and $0.01 \mathrm{hPa}$ from November to January. When the model lid is lowered the temperature response is degraded, even when momentum is conserved. In particular, the maximum negative temperature response increases and the dynamical warming response weakens. This is consistent with the biases seen in the control climate for this configuration. Nonconservation further degrades the response to ozone depletion; the cooling is even larger and the dynamical warming is weakened further. Thus, momentum conservation leads to a temperature response to ozone depletion that is in better agreement with the truth, but cannot entirely compensate for the problems associated with a 10-hPa lid.

In conjunction with the cooling response in the high configuration there is an eastward zonal wind response to ozone depletion. Lowering the model lid while conserving momentum results in a larger eastward response above $100 \mathrm{hPa}$, but good agreement below $100 \mathrm{hPa}$. In particular, the zonal wind response near the surface is unaltered. However, when momentum is not conserved the response is degraded from $10 \mathrm{hPa}$ to the surface; in particular, the response above $100 \mathrm{hPa}$ is even stronger and the extension to the surface is less significant. The impact of momentum conservation on the surface response to ozone depletion is apparent in mean sea level pressure differences. The high configuration has a negative annular mean sea level pressure response to ozone depletion over the polar cap, which is in good agreement with observations. When the lid is lowered and momentum is conserved this response is mostly preserved with the exception of a spurious positive response equatorward of $60^{\circ} \mathrm{S}$. This spurious response is reminiscent of that seen in the control climate in the Antarctic when the lid is lowered (Fig. 10), and it may reflect the same sort of planetary wave errors because the ozone hole perturbation implies a further strengthening of the already strong Antarctic jet, such that the bias seen in the control in the Antarctic is further exacerbated by the perturbation. However, when momentum is not conserved the surface response to ozone depletion is significantly weakened and is less annular at the pole.

This impact of momentum conservation on the surface response to ozone depletion in the lowered configuration has implications for low-lid models that try to simulate the tropospheric response to ozone depletion and recovery, 
as were used in the latest Intergovernmental Panel on Climate Change (IPCC) assessment report (Solomon et al. 2007). Perlwitz et al. (2008) have shown that the models with imposed ozone recovery used in Solomon et al. (2007) do not capture the weakening of the surface southern annular mode that would be expected to arise from ozone recovery. Our results suggest that for low-lid models, the modeled response to ozone depletion and recovery is sensitive to whether or not gravity wave momentum flux is conserved and to model-lid height. In particular, low-lid models that do not conserve momentum would be expected to have a much weaker surface response to ozone depletion relative to low-lid models that do conserve momentum, which themselves have a slightly distorted response relative to the response in high-lid models.

The dynamical response to ozone depletion in the high configuration in December and January, as measured by changes in the vertical residual circulation, is in good agreement with previous modeling studies. The main effect of lowering the model lid (while conserving momentum) on the dynamical response is a delay of the EPFD-induced downwelling, which is attributed to biases in the resolved waves in the control climate. Enforcing conservation of momentum in the lowered configuration ensures that the GWD-induced downwelling response is not significantly altered when the lid is lowered to $10 \mathrm{hPa}$. The GWD-induced downwelling has roughly equal contributions from the orographic and nonorographic components. In contrast, when momentum is not conserved there is virtually no GWD-induced downwelling, and the EPFD-induced downwelling response is completely distorted. In fact, there is then no statistically significant gravity wave or planetary wave response to ozone depletion from September to December. Thus, conservation of momentum in the lowered configuration ensures the physically correct gravity wave response, and through the interaction with the mean flow substantially improves the planetary wave response. The sensitivity of the parameterized response to whether or not momentum is conserved is in agreement with the results of SS07. Allowing momentum flux to escape to space, as occurs in the nonconservative configuration, leads to missing downwelling, and hence a weakened dynamical response. However, it is clear that even with momentum conservation, models with a midstratospheric lid cannot correctly represent the dynamics of the Antarctic polar vortex.

Conservation of parameterized momentum flux should be enforced in all numerical implementations of GWD parameterizations (both orographic and nonorographic) to avoid introducing spurious biases in the modeled climate and its response to climate perturbations. Here it was shown that nonconservation always leads to detrimental effects on the high-latitude climate and its re- sponse to ozone depletion. The biases resulting from nonconservation would, in practice, require further (and completely unnecessary) tuning to compensate for them. Furthermore, momentum conservation reduces the sensitivity of modeled climate to model-lid height; the differences between the high and lowered configurations were always significantly larger when momentum was not conserved. Such nonrobustness leads to illposed high-low-lid model comparisons. Model-lid height comparisons are currently a key tool used in assessing the impact of the stratosphere on tropospheric climate (Shaw and Shepherd 2008) and they should focus on robust responses to changes in model-lid height.

Acknowledgments. This research has been supported by the Natural Sciences and Engineering Research Council of Canada, in part through a Canada Graduate Scholarship to the first author, and by the Canadian Foundation for Climate and Atmospheric Sciences. The first author also acknowledges support from the Canadian Meteorological and Oceanographic Society and Zonta International. The first author is grateful to Dr. Charles McLandress for assistance with the residual vertical velocity calculations and for many helpful discussions. The authors also thank two anonymous reviewers whose comments helped to improve the submitted manuscript.

\section{REFERENCES}

Andrews, D. G., J. R. Holton, and C. B. Leovy, 1987: Middle Atmosphere Dynamics. Academic Press, 489 pp.

Boville, B. A., and X. Cheng, 1988: Upper boundary effects in a general circulation model. J. Atmos. Sci., 45, 2591-2606.

Eyring, V., and Coauthors, 2006: Assessment of temperature, trace species, and ozone in chemistry-climate model simulations of the recent past. J. Geophys. Res., 111, D22308, doi:10.1029/ 2006JD007327.

Garcia, R. R., and B. A. Boville, 1994: "Downward control" of the mean meridional circulation and temperature distribution of the polar winter stratosphere. J. Atmos. Sci., 51, 2238-2245.

Haynes, P. H., C. J. Marks, M. E. McIntyre, T. G. Shepherd, and K. P. Shine, 1991: On the "downward control" of extratropical diabatic circulations by eddy-induced mean zonal forces. J. Atmos. Sci., 48, 651-678.

Hitchcock, P., T. G. Shepherd, and C. McLandress, 2009: Past and future conditions for polar stratospheric cloud formation simulated by the Canadian Middle Atmosphere Model. Atmos. Chem. Phys., 9, 483-495.

Holton, J. R., 1983: The influence of gravity wave breaking on the general circulation of the middle atmosphere. J. Atmos. Sci., 40, 2497-2507.

Lawrence, B. A., 1997: Some aspects of the sensitivity of stratospheric climate simulations to model lid height. J. Geophys. Res., 102, 23 805-23 811.

Mahlman, J. D., L. J. Umscheid, and J. P. Pinto, 1994: Transport, radiative, and dynamical effects of the Antarctic ozone hole: A GFDL "SKYHI" model experiment. J. Atmos. Sci., 51, $489-508$ 
Manzini, E., B. Steil, C. Brühl, M. A. Giorgetta, and K. Krüger, 2003: A new interactive chemistry-climate model: 2 . Sensitivity of the middle atmosphere to ozone depletion and increase in greenhouse gases and implications for recent stratospheric cooling. J. Geophys. Res., 108, 4429, doi:10.1029/2002JD002977.

McFarlane, N. A., 1987: The effect of orographically excited gravity wave drag on the circulation of the lower stratosphere and troposphere. J. Atmos. Sci., 44, 1775-1800.

McLandress, C., and N. A. McFarlane, 1993: Interactions between orographic gravity wave drag and forced stationary planetary waves in the winter Northern Hemisphere middle atmosphere. J. Atmos. Sci., 50, 1966-1990.

_ in the Brewer-Dobson circulation, including its extension to high latitudes. J. Climate, 22, 1516-1540.

Newman, P. A., E. R. Nash, and J. E. Rosenfield, 2001: What controls the temperature in the Arctic stratosphere in the spring? J. Geophys. Res., 106, 19 999-20 010.

Perlwitz, J., S. Pawson, R. L. Fogt, J. E. Nielsen, and W. D. Neff, 2008: The impact of stratospheric ozone hole recovery on Antarctic climate. Geophys. Res. Lett., 35, L08714, doi:10.1029/ 2008 GL033317.

Randel, W. J., and F. Wu, 1999: Cooling of the Arctic and Antarctic polar stratospheres due to ozone depletion. J. Climate, 12, 1467-1479.

Scinocca, J. F., 2003: An accurate spectral non-orographic gravity wave parameterization for general circulation models. $J$. Atmos. Sci., 60, 667-682.
, and N. A. McFarlane, 2000: The parameterization of drag induced by stratified flow over anisotropic topography. Quart. J. Roy. Meteor. Soc., 126, 2353-2393.

— - - M. Lazare, and D. Plummer, 2008: The CCCma third generation AGCM and its extension into the middle atmosphere. Atmos. Chem. Phys., 8, 7055-7074.

Shaw, T. A., and T. G. Shepherd, 2007: Angular momentum conservation and gravity wave drag parameterization: Implications for climate models. J. Atmos. Sci., 64, 190-203.

—, and - 2008: Raising the roof. Nat. Geosci., 1, 12-13.

Shepherd, T. G., and T. A. Shaw, 2004: The angular momentum constraint on climate sensitivity and downward influence in the middle atmosphere. J. Atmos. Sci., 61, 2899-2908.

— backs in middle-atmosphere models. J. Geophys. Res., 101, 23 447-23 464.

Sigmond, M., J. F. Scinocca, and P. J. Kushner, 2008: Impact of the stratosphere on tropospheric climate change. Geophys. Res. Lett., 35, L12706, doi:10.1029/2008GL033573.

Solomon, S., D. Qin, M. Manning, M. Marquis, K. Averyt, M. Tignor, H. L. Miller Jr., and Z. Chen, Eds., 2007: Climate Change 2007: The Scientific Basis. Cambridge University Press, 996 pp.

Thompson, D. W. J., and S. Solomon, 2002: Interpretation of recent Southern Hemisphere climate change. Science, 296, 895899. 